\title{
Factors Associated With Upper Extremity Motor Recovery After Repetitive Transcranial Magnetic Stimulation in Stroke Patients
}

\author{
Jong Hwa Lee, $\mathrm{MD}^{1,2}$, Sang Beom Kim, MD ${ }^{1,2}$, Kyeong Woo Lee, $\mathrm{MD}^{1,2}$, \\ Min Ah Kim, $\mathrm{MD}^{1,2}$, Sook Joung Lee, $\mathrm{MD}^{1,2}$, Su Jin Choi, $\mathrm{MD}^{1,2}$ \\ ${ }^{1}$ Department of Physical Medicine \& Rehabilitation, Dong-A University College of Medicine, Busan; \\ ${ }^{2}$ Regional Cardiocerebrovascular Center, Dong-A University Hospital, Busan, Korea
}

Objective To determine factors associated with motor recovery of the upper extremity after repetitive transcranial magnetic stimulation (rTMS) treatment in stroke patients.

Methods Twenty-nine patients with subacute stroke participated in this study. rTMS was applied to the hand motor cortex for 10 minutes at a $110 \%$ resting motor threshold and $10 \mathrm{~Hz}$ frequency for two weeks. We evaluated the biographical, neurological, clinical, and functional variables, in addition to the motor-evoked potential (MEP) response. The Manual Function Test (MFT) was performed before, immediately after, and two weeks after, the treatment. Patients were divided into a responder and non-responder group according to their respective improvements on the MFT. Data were compared between the two groups.

Results Patients with exclusively subcortical stroke, absence of aphasia, the presence of a MEP response, high scores on the Mini-Mental Status Examination, Motricity Index arm score, Functional Independence Measure, and Functional Ambulatory Classification; and a shorter period from stroke onset to rTMS were found to be significantly associated with a response to rTMS.

Conclusion The results of this study suggest that rTMS may have a greater effect on upper extremity motor recovery in stroke patients who have a MEP response, suffer an exclusively subcortical stroke, mild paresis, and have good functional status. Applying rTMS early would have additional positive effects in the patients with the identified characteristics.

Keywords Stroke, Transcranial magnetic stimulation, Motor function

Received April 14, 2014; Accepted October 7, 2014

Corresponding author: Su Jin Choi

Department of Physical Medicine and Rehabilitation, Dong-A University Hospital, 26 Daesingongwon-ro, Seo-gu, Busan 602-715, Korea

Tel: +82-51-240-5690, Fax: +82-51-254-8511, E-mail: mail1002zoo@hanmail.net

(a) This is an open-access article distributed under the terms of the Creative Commons Attribution Non-Commercial License (http://creativecommons.org/ licenses/by-nc/3.0) which permits unrestricted noncommercial use, distribution, and reproduction in any medium, provided the original work is properly cited. Copyright $\odot 2015$ by Korean Academy of Rehabilitation Medicine 


\section{INTRODUCTION}

Repetitive transcranial magnetic stimulation (rTMS) applied to the affected hemisphere is considered an effective approach that contributes to motor recovery of the upper extremity [1,2] not only in motor function [3-5], but also in motor power [6] after stroke. It is believed that the effect of rTMS is related to changes in corticomotor excitability [5-7].

Unfortunately, not all stroke survivors benefit from rTMS. To achieve the most efficient use of rTMS, it is important to identify the factors associated with a beneficial response to rTMS. However, very few studies have been conducted to identify the predictors of high-frequency rTMS response. Ameli et al. [3] proposed that neural activity in ipsilesional M1 may serve as a surrogate marker for the effectiveness of facilitatory rTMS. On the other hand, Emara et al. [8] suggested that patients with cortical strokes experience less improvement when compared to those with subcortical strokes with inhibitory, but not facilitatory, rTMS. The authors reported that the patients with total anterior circulation strokes showed no significant improvement. The purpose of the present study is to determine the factors associated with recovery of upper extremity motor function after rTMS treatment in stroke patients.

\section{MATERIALS AND METHODS}

A total of 29 patients were included in this study. Inclusion criteria were predefined as follows: 1 ) onset of symptoms at least three months before recruitment; 2) hemispheric infarction; 3) first onset stroke; and 4) decreased motor power of the upper extremity. Exclusion criteria were 1) bilateral cerebrovascular disease; 2) implanted pacemaker or medication pump, metal plate in skull, or metal objects in the eyes or skull; 3) craniectomy state; and 4) inability to cooperate due to severe aphasia or agnosia.

Before performing rTMS, we evaluated the motorevoked potentials (MEPs) of the abductor pollicis brevis (APB) muscle on the lesion side using a MagPro (MagVenture Inc., Farum, Denmark). All participants were seated comfortably in chairs. Surface electromyographic electrodes were placed over the APB muscle on the lesion side and connected to a Nicolet Electromyo- graph (Biomedical Inc., Madison, WI, USA). Magnetic stimulation using a circular coil (external diameter, 14 $\mathrm{cm}$ ) was delivered on the scalp over the affected hemisphere, moving on a grid of approximately 1 by $1 \mathrm{~cm}$ in order to find the hotspot for eliciting MEP in the APB muscle. The hotspot is the spot where the MEPs with larger amplitudes were evoked with minimal stimulation intensity. The resting motor threshold (rMT) was defined as the lowest stimulus intensity eliciting MEPs $\geq 50 \mu \mathrm{V}$ in at least five of 10 consecutive stimulations.

The MagPro instrument was used to deliver stimulatory trains using a figure-eight coil cooled with air. rTMS was performed on the ipsilesional hemisphere hotspot at $110 \%$ of each rMT, at $10 \mathrm{~Hz}$, for 10 seconds, and repeated every minute for 10 minutes (100 stimuli $\times 10$ times; interstimulus interval, 50 seconds). rTMS was administered five days per week for two weeks (10 treatments in total).

Our primary outcome measure was the Manual Function Test (MFT) of the affected upper extremity. The MFT is an index for assessing motor function disorders in patients' paralyzed upper extremity from stroke. Eight tasks in the following three categories were performed using standardized equipment: 'arm motions,' 'grasp and pinch', and 'arm and hand activities'. The maximum possible value for the total score was 32 [9]. This outcome measure was obtained before, immediately after, and two weeks after, rTMS treatment.

Clinical variables included the biographical (age, sex) characteristics of the patient, the neurological characteristics (site and extent of the lesion, period from stroke onset to rTMS), and the clinical characteristics (National Institutes of Health Stroke Scale [NIHSS], presence of aphasia, Mini-Mental Status Examination [MMSE], Motricity Index arm and leg scores [MI-A and MI-L], and Modified Ashworth Scales in the elbow flexor, elbow extensor, and finger flexor muscles of the affected upper extremity). Functional variables included the MFT, Functional Independence Measure (FIM), the Korean version of the Modified Barthel Index (K-MBI) and Functional Ambulatory Category (FAC).

To estimate the extent of the lesion, all included patients underwent MRI (1.5T Signa EchoSpeed superconducting imaging system; GE Medical Systems, Milwaukee, WI, USA). The diffusion-weighted imaging (DWI) lesion volume was determined by manually tracing the edge of the hyperintense signal on each slice of the trace 
DWI scans. The area of a region of interest was multiplied by the section thickness plus the intersection gap and then totaled to give the lesion volume.

The FIM is a tool used to assess performance during tasks that can broadly be categorized as activities of daily living (ADL), mobility, and cognition. It has a total of 18 items. Each item is scored from 1 to 7 , with 7 signifying complete independence or normal functioning, and 1 signifying complete dependence or requiring total assistance. The total maximum score for FIM is 126, which implies total independence. The minimum score is 18 , which implies the provision of full assistance in all 18 items assessed [10]. The Barthel Index also measures independence in ADL; the maximum score is 100 . The 10 ADLs assessed are bowel control, bladder control, personal hygiene, toilet transfer, bathtub transfer, feeding, dressing, wheelchair transfer to and from bed, walking (wheelchair management, if the patient is non-ambulatory), and ascending and descending stairs [11]. We administered the K-MBI, which was developed in 2006, by translating the 5th version of the MBI [12].

Responders were defined as patients who achieved a minimal clinically important difference (MCID) in terms of MFT improvement. We calculated MCID using distribution-based methods (half of the standard deviation) [13]. Patients were classified into two groups according to their improvements in MFT either immediately after or two weeks after rTMS, a responder group and a nonresponder group (Fig. 1).

For the statistical analysis, SPSS software ver. 12.0 for Windows (SPSS Inc., Chicago, IL, USA) was used. The chisquare test was used to analyze differences in categorical variables in the responder and non-responder groups. The Mann-Whitney U test was used to evaluate differences in continuous variables between the two groups and compare improvements in MFT depending on the categorical variables. Because there were large deviations in the extent of the lesion, we divided the patients into three groups based on lesion area (group 1, $<50 \mathrm{~cm}^{2}$; group $2, \geq 50 \mathrm{~cm}^{2}$ and $<100 \mathrm{~cm}^{2}$; group $3, \geq 100 \mathrm{~cm}^{2}$ ). The Kruskal-Wallis test was used to compare the three groups of improvements with the MFT. The Pearson correlation analysis was used to identify significant correlations between continuous variables and improvements in MFT. Multivariate regression analysis in a forward stepwise manner was used to distinguish variables affecting improvements in the MFT immediately after and two weeks after treatment. Categorical variables were transformed into dummy variables. Statistical significance was accepted for $\mathrm{p}$-values of $<0.05$.

\section{RESULTS}

A total of 29 patients were included in this study, 20
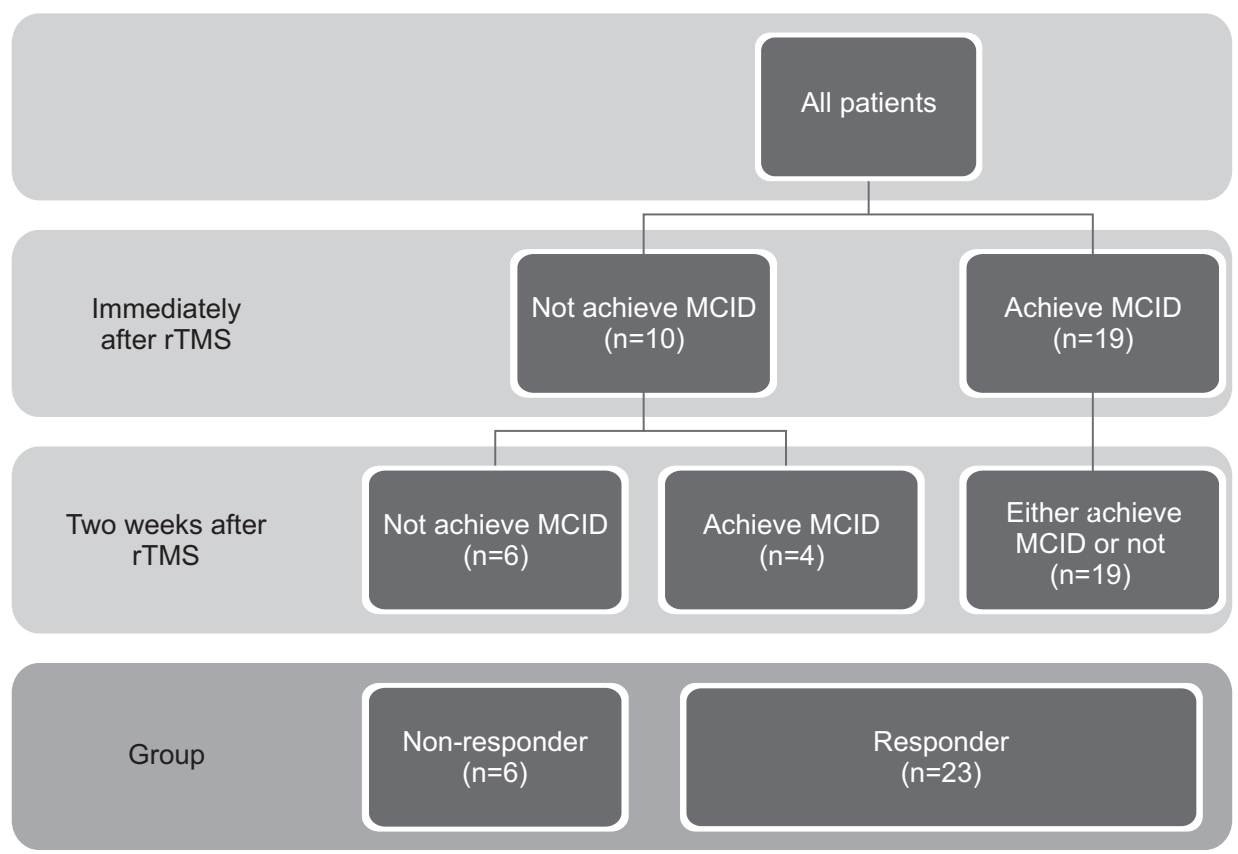

Fig. 1. Patients were classified into two groups according to their improvements in Manual Function Test either immediately after or two weeks after rTMS. MCID, minimal clinically important difference; rTMS, repetitive transcranial magnetic stimulation. 
men $(69.0 \%)$ and nine women (31\%). We compared patients who achieved MCID either immediately after or two weeks after rTMS (responders) with patients who did not achieve MCID at all (non-responders) for potential variables (Table 1). Twenty-three patients (79.3\%) were in the responder group and six patients $(20.7 \%)$ were in the non-responder group. Age and sex factors were not significantly different between the two groups. The incidence of exclusively subcortical infarction was higher in the responder group, although the extent of the lesion was not significantly different between the two groups. The period from stroke onset to rTMS was shorter in the responder group. NIHSS was not significantly different between the two groups. Aphasia was more common, and MMSE and MI-A were higher, in the responder group. MI-L and the incidence of spasticity were not different between the two groups. A MEP response was more likely to occur in the responder group. MFT and K-MBI tended to be higher in the responder group, although this finding did not reach statistical significance. FIM and FAC were significantly higher in the responder group.

Among patients who achieved MCID immediately after rTMS, 14 patients (73.7\%) achieved MCID two weeks after rTMS and five patients $(26.3 \%)$ did not. Patients who

Table 1. Comparison of patients who achieved MCID either immediately after or two weeks after rTMS (responders) with patients who did not achieve MCID at all (non-responders) for potential variables

\begin{tabular}{|c|c|c|c|}
\hline Variable & Responder $(n=23)$ & Non-responder $(n=6)$ & p-value $e^{a)}$ \\
\hline Age (yr) & $65.5 \pm 10.8$ & $69.5 \pm 7.8$ & 0.250 \\
\hline Sex (male:female) & $15: 8$ & $5: 1$ & 0.360 \\
\hline \multicolumn{4}{|l|}{ Site of the lesion } \\
\hline Right:left & $13: 10$ & $1: 5$ & 0.099 \\
\hline Cortical+subcortical: subcortical & $12: 11$ & $6: 0$ & 0.026 \\
\hline Extent of the lesion $\left(\mathrm{cm}^{2}\right)$ & $18.8 \pm 22.6$ & $216.8 \pm 296.4$ & 0.730 \\
\hline Period from stroke onset to rTMS (day) & $21.5 \pm 15.3$ & $41.7 \pm 19.5$ & 0.019 \\
\hline NIHSS & $6.7 \pm 4.1$ & $11.0 \pm 7.0$ & 0.260 \\
\hline Aphasia (-:+) & $17: 6$ & $2: 4$ & 0.047 \\
\hline MMSE & $22.1 \pm 7.7$ & $7.3 \pm 10.3$ & 0.003 \\
\hline \multicolumn{4}{|l|}{ MI } \\
\hline Arm score & $48.4 \pm 24.9$ & $16.3 \pm 24.2$ & 0.014 \\
\hline Leg score & $60.9 \pm 22.3$ & $42.3 \pm 28.4$ & 0.068 \\
\hline \multicolumn{4}{|l|}{ MAS (-:+) } \\
\hline $\mathrm{EF}$ & $22: 1$ & $6: 0$ & 0.138 \\
\hline $\mathrm{EE}$ & $20: 3$ & $6: 0$ & 0.214 \\
\hline Finger flexor & $22: 1$ & $5: 1$ & 0.183 \\
\hline $\operatorname{MEP}(-:+)$ & $1: 22$ & $4: 2$ & 0.001 \\
\hline MFT & $13.0 \pm 10.1$ & $5.7 \pm 10.5$ & 0.052 \\
\hline FIM & $65.5 \pm 22.3$ & $39.2 \pm 24.2$ & 0.033 \\
\hline K-MBI & $41.3 \pm 27.1$ & $19.5 \pm 27.7$ & 0.073 \\
\hline FAC & $0.8 \pm 1.0$ & $0.0 \pm 0.0$ & 0.035 \\
\hline
\end{tabular}

Values are presented as mean \pm standard deviation or number.

MCID, minimal clinically important difference; rTMS, repetitive transcranial magnetic stimulation; NIHSS, National Institutes of Health Stroke Scale; (-), absence; (+), presence; MMSE, Mini-Mental Status Examination; MI, side score of Motricity Index; MAS, Modified Ashworth Scale; EF, elbow flexor; EE, elbow extensor; MEP, motor-evoked potential; MFT, Manual Function Test; FIM, Functional Independence Measure; K-MBI, Korean version of the Modified Barthel Index; FAC, Functional Ambulatory Classification.

a) -values were calculated using the Mann-Whitney $U$ test for continuous variables and the chi-square test for categorical variables. 
were responders immediately after rTMS, but not two weeks after rTMS, showed higher MFT than patients who were responders both immediately after and two weeks after rTMS (Table 2).

Among patients who were non-responders immediately after rTMS, six patients (60\%) achieved MCID two weeks after rTMS and four patients (40\%) did not. All of the patients who were non-responders both immediately after and two weeks after rTMS, had cortical and subcortical infarction, a longer period from stroke onset to rTMS, and lower MMSE (Table 2).

Table 3 summarizes the relationships between the clinical factors and improvements in MFT immediately after rTMS. A Mann-Whitney U test showed that patients with extensively subcortical strokes and MEP responses achieved greater improvements in MFT. A Kruskal-Wallis test showed that patients with a larger lesion achieved greater improvements in MFT. Pearson correlation analysis showed a significant correlation between improvements in MFT immediately after rTMS and MMSE.

The same analyses were repeated for MFT improvements two weeks after rTMS. A Mann-Whitney U test showed that greater MFT improvements were achieved in patients with left-hemispheric cerebral infarction, apha-

Table 2. Characteristics of patients who showed different responses immediately after rTMS and two weeks after rTMS

\begin{tabular}{|c|c|c|c|c|}
\hline \multirow[b]{2}{*}{ Variable } & \multicolumn{2}{|c|}{ Achieved MCID $^{a)}(n=19)$} & \multicolumn{2}{|c|}{ Did not achieve MCID $^{a}(n=10)$} \\
\hline & $\begin{array}{c}\text { Achieved } \\
\operatorname{MCID}^{b)}(n=14)\end{array}$ & $\begin{array}{c}\text { Did not achieve } \\
\operatorname{MCID}^{\text {b) }}(n=5)\end{array}$ & $\begin{array}{c}\text { Achieved } \\
\operatorname{MCID}^{b)}(n=4)\end{array}$ & $\begin{array}{c}\text { Did not achieve } \\
\operatorname{MCID}^{b)}(n=6)\end{array}$ \\
\hline Age (yr) & $65.3 \pm 9.3$ & $66.0 \pm 16.1$ & $66.1 \pm 10.2$ & $69.5 \pm 7.8$ \\
\hline Sex (male:female) & 9:5 & $3: 2$ & $3: 1$ & $5: 1$ \\
\hline \multicolumn{5}{|l|}{ Site of the lesion } \\
\hline Right:left & 9:5 & $1: 4$ & 3:1 & 1:5 \\
\hline Cortical+subcortical:subcortical & $7: 7$ & $3: 2$ & $2: 2^{\mathrm{d})}$ & $6: 0$ \\
\hline Extent of the lesion $\left(\mathrm{cm}^{2}\right)$ & $22.7 \pm 26.3$ & $113.0 \pm 10.6$ & $12.8 \pm 18.4$ & $216.8 \pm 296.4$ \\
\hline Period from stroke onset to rTMS (day) & $25.6 \pm 17.9$ & $15.4 \pm 3.8$ & $13.0 \pm 3.5^{\mathrm{c})}$ & $41.7 \pm 19.5$ \\
\hline NIHSS & $7.4 \pm 4.9$ & $5.2 \pm 1.6$ & $5.7 \pm 5.1$ & $11.0 \pm 7.0$ \\
\hline Aphasia $(-:+)$ & $11: 3$ & $3: 2$ & 3:1 & $2: 4$ \\
\hline MMSE & $22.1 \pm 8.4$ & $21.6 \pm 9.0$ & $23.0 \pm 1.7^{\mathrm{c})}$ & $7.3 \pm 10.2$ \\
\hline \multicolumn{5}{|l|}{ MI } \\
\hline Arm score & $45.6 \pm 27.0$ & $61.8 \pm 19.3$ & $38.7 \pm 19.6$ & $16.3 \pm 24.2$ \\
\hline Leg score & $55.9 \pm 24.9$ & $72.0 \pm 15.0$ & $65.7 \pm 14.2$ & $42.3 \pm 28.4$ \\
\hline \multicolumn{5}{|l|}{ MAS (-:+) } \\
\hline $\mathrm{EF}$ & 13:1 & $5: 0$ & $4: 0$ & $6: 0$ \\
\hline $\mathrm{EE}$ & 13:1 & $4: 1$ & $3: 1$ & $6: 0$ \\
\hline Finger flexor & 13:1 & $5: 0$ & $4: 0$ & $5: 1$ \\
\hline MEP (-:+) & $14: 0$ & $4: 1$ & $0: 4$ & $4: 2$ \\
\hline MFT & $10.6 \pm 8.7^{\mathrm{c})}$ & $22.2 \pm 11.9$ & $8.7 \pm 3.8$ & $5.7 \pm 10.5$ \\
\hline FIM & $58.1 \pm 22.3$ & $83.2 \pm 15.2$ & $70.7 \pm 17.8$ & $39.2 \pm 24.2$ \\
\hline K-MBI & $31.9 \pm 25.6$ & $69.0 \pm 15.9$ & $39.0 \pm 20.5$ & $19.5 \pm 27.7$ \\
\hline FAC & $0.7 \pm 1.1$ & $1.4 \pm 0.9$ & $0.3 \pm 0.6$ & $0.0 \pm 0.0$ \\
\hline
\end{tabular}

Values are presented as mean \pm standard deviation or number.

MCID, minimal clinically important difference; rTMS, repetitive transcranial magnetic stimulation; NIHSS, National Institutes of Health Stroke Scale; (-), absence; (+), presence; MMSE, Mini-Mental Status Examination; MI, side score of Motricity Index; MAS, Modified Ashworth Scale; EF, elbow flexor; EE, elbow extensor; MEP, motor-evoked potential; MFT, Manual Function Test; FIM, Functional Independence Measure; K-MBI, Korean version of the Modified Barthel Index; FAC, Functional Ambulatory Classification.

${ }^{a)}$ Immediately after rTMS, ${ }^{\text {b) }}$ two weeks after rTMS, ${ }^{c)} \mathrm{p}<0.05$ by Mann-Whitney $\mathrm{U}$ test, ${ }^{\mathrm{d})} \mathrm{p}<0.05$ by chi-square test. 
Table 3. Relationship between clinical variables and improvement of MFT at each follow-up

\begin{tabular}{|c|c|c|c|c|}
\hline \multirow{2}{*}{ Variable } & \multicolumn{2}{|c|}{ Immediately after rTMS } & \multicolumn{2}{|c|}{ Two weeks after rTMS } \\
\hline & p-value ${ }^{\text {a) }}$ & $\mathbf{r}^{\mathbf{b})}$ & p-value ${ }^{\text {a) }}$ & $\mathbf{r}^{\mathbf{b})}$ \\
\hline Age (yr) & 0.163 & -0.217 & 0.282 & -0.210 \\
\hline Sex (male:female) & 0.355 & & 0.128 & \\
\hline \multicolumn{5}{|l|}{ Site of the lesion } \\
\hline Right:left & 0.486 & & 0.011 & \\
\hline Cortical+subcortical:subcortical & 0.006 & & 0.061 & \\
\hline Extent of the lesion $\left(\mathrm{cm}^{2}\right)$ & 0.040 & & 0.029 & \\
\hline Period from stroke onset to rTMS (day) & 0.716 & -0.072 & 0.482 & -0.138 \\
\hline NIHSS & 0.196 & -0.252 & 0.695 & -0.078 \\
\hline Aphasia $(-:+)$ & 0.162 & & 0.037 & \\
\hline MMSE & 0.006 & 0.510 & 0.014 & 0.459 \\
\hline \multicolumn{5}{|l|}{ MI } \\
\hline Arm score & 0.264 & 0.218 & 0.412 & 0.161 \\
\hline Leg score & 0.246 & 0.227 & 0.736 & 0.067 \\
\hline \multicolumn{5}{|l|}{ MAS (-:+) } \\
\hline $\mathrm{EF}$ & 0.710 & & 0.552 & \\
\hline $\mathrm{EE}$ & 0.734 & & 0.573 & \\
\hline Finger flexor & 0.684 & & 0.549 & \\
\hline MEP (-:+) & 0.006 & & 0.004 & \\
\hline MFT & 0.950 & 0.012 & 0.697 & -0.077 \\
\hline FIM & 0.106 & 0.312 & 0.734 & 0.067 \\
\hline K-MBI & 0.302 & 0.202 & 0.582 & -0.109 \\
\hline FAC & 0.284 & 0.210 & 0.639 & 0.093 \\
\hline
\end{tabular}

rTMS, repetitive transcranial magnetic stimulation; NIHSS, National Institutes of Health Stroke Scale; (-), absence; (+), presence; MMSE, Mini-Mental Status Examination; MI, side score of Motricity Index; MAS, Modified Ashworth Scale; EF, elbow flexor; EE, elbow extensor; MEP, motor-evoked potential; MFT, Manual Function Test; FIM, Functional Independence Measure; K-MBI, Korean version of the Modified Barthel Index; FAC, Functional Ambulatory Classification.

${ }^{a)} \mathrm{p}$-values were calculated using the correlation analysis for continuous variables and the Mann-Whitney $\mathrm{U}$ test for categorical variables except for extent of the lesion, for which the Kruskal-Wallis test was used, ${ }^{\mathrm{b})}$ Pearson correlation.

Table 4. Multivariate regression analysis with improvements in MFT at each follow-up

\begin{tabular}{ccccccc}
\hline \multirow{2}{*}{ Variable } & \multicolumn{2}{c}{$\begin{array}{c}\text { Immediately after } \\
\text { rTMS }\left(\mathbf{R}^{2}=\mathbf{0 . 2 0 2}\right)\end{array}$} & & \multicolumn{2}{c}{$\begin{array}{c}\text { Two weeks after } \\
\text { rTMS }\left(\mathbf{R}^{2}=\mathbf{0 . 2 1 5}\right)\end{array}$} \\
\cline { 2 - 3 } \cline { 5 - 6 } & $\beta$ & $\mathbf{p}$-value & & $\beta$ & p-value \\
\hline MEP & 0.481 & 0.010 & & 0.494 & 0.008 \\
\hline
\end{tabular}

MFT, Manual Function Test; rTMS, repetitive transcranial magnetic stimulation; MEP, motor-evoked potential; $\mathrm{R}^{2}$, R-square.

sia, and a MEP response (Table 3). A Kruskal-Wallis test showed that patients with larger lesions achieved greater MFT improvements. A Pearson correlation analysis showed a significant correlation between improvements in MFT and MMSE.

According to multivariate regression analysis, only the presence of a MEP response was significantly associated with the effect of rTMS immediately after and two weeks after treatment $(\mathrm{p}<0.05)$, although the explanatory power was low (immediately after rTMS, $\mathrm{R}^{2}=0.202$; two weeks after rTMS, $\mathrm{R}^{2}=0.224$ ) (Table 4).

\section{DISCUSSION}

This study was designed to investigate the factors associated with responses to high-frequency $(10 \mathrm{~Hz})$ rTMS 
applied over ipsilesional M1 in patients who had subacute strokes. We showed that facilitatory rTMS causes more improvement in patients with exclusive subcortical lesions, a MEP response, stronger motor power, higher cognitive and ambulatory function, more independence in $\mathrm{ADL}$, and without aphasia before rTMS treatment.

In this study, immediately after rTMS, patients with higher cognitive function, stronger motor power, the presence of MEP, and higher ambulatory function responded better to high-frequency rTMS. At two weeks after rTMS, patients with right-hemispheric cerebral infarction a MEP response, and without aphasia showed more benefits from rTMS. About one-third of patients who responded immediately after rTMS did not respond two weeks after rTMS. As they had stronger motor power and higher affected upper extremity function, this result might be a ceiling effect. About half of the patients who were not responders immediately after rTMS showed a response two weeks after rTMS. These patients showed a higher incidence of exclusively subcortical infarction, higher cognitive function, and shorter periods from stroke onset to rTMS than patients who did not respond at all. Although these findings showed no statistical significance, all of the patients who responded two weeks after rTMS had a MEP response and only one-third of the patients who did not respond to rTMS did.

In this study, all of the patients who did not respond at all had cortical and subcortical infarctions. Ameli et al. [3] showed that patients with subcortical infarctions showed improvement in movement kinematics, whereas the cortical patients showed either no change or, in some cases, worsening in movement kinematics with high-frequency rTMS $(10 \mathrm{~Hz})$. The authors postulated that this difference may be due to the loss of excitable neural tissue, a change of neural connectivity between ipsilesional $\mathrm{Ml}$ and the cortical motor network, and a loss of GABAergic intracortical inhibition, causing an enhancement of glutamatergic activity.

Our study also showed that patients with aphasia received less benefit from rTMS. Since all of the patients with aphasia had cortical and subcortical stroke, it might be related to the effect of the site of the lesion.

In this study, patients with lower cognitive function did not respond to high-frequency rTMS. Among the patients with lower cognitive function, about half of them responded to rTMS treatment. All of the patients who responded to rTMS showed a MEP response, stronger motor power, and higher function in the affected upper extremity. This finding shows that other variables, such as the presence of MEP and affected side upper extremity baseline motor power were more significant than the patient's cognitive function.

An important result from the present investigation is that the response to rTMS is strongly associated with the presence of a MEP response (positive predictive value, $91.7 \%$; negative predictive value, $80 \%$ ). MEPs, or their absence, provide indicators of the functional integrity and excitability of the corticomotor pathway [14]. Given that the capacity for rTMS to influence cortical excitability contributes to the rationale for its use as a therapeutic adjuvant after stroke, if there is excitability of the corticomotor pathway, the effect of rTMS would be magnified [15]. Arm and leg paresis are easy to determine and could also be used to investigate the integrity of the corticomotor pathway. The residual strength of the paretic muscle could be a useful predictor of response to rTMS treatment.

Our results show that response to rTMS treatment increases when rTMS is applied earlier. Because we administered rTMS when the patient was medically stable and could sit in a wheelchair for 10 minutes, severely impaired patients tended to have a longer period from stroke onset to rTMS. Another explanation for this finding could be different mechanisms of rTMS according to stroke duration. Lefaucheur [16] reported that, in chronic stroke, the application of rTMS is meant to recruit or activate compensatory pathways and promote adaptive plasticity. In contrast, rTMS applied in the acute phase of stroke is meant to limit neuronal loss. Further studies should address how the interval between the stroke onset and rTMS influences its efficacy.

We found that patients who responded to rTMS showed greater improvements in independence in ADL, but not in ambulatory function. Independence in ADL largely depends on upper extremity function, while ambulatory function depends on lower extremity function. Thus, it seems that rTMS over the motor cortex of the APB muscle could not directly contribute to improvements in lower extremity function.

We used a circular coil to evaluate the MEPs and find hotspots. Reliability is better with the figure-eight coil, presumably due to its more focused stimulation [17]. 
We used a circular coil because it is easy to evaluate the MEPs, and thus patients feel less discomfort. This is due to the higher corticospinal excitability of the circular coil. Although Fleming et al. [18] found poor reliability of MEP amplitude at $120 \%$ rMT for the circular coil, they reported moderate to good reliability of rMT for the same coil. Malcolm et al. [19] reported good stimulus-response curve repeatability for APB with the circular coil, though they made no direct comparison with the figure-eight coil. We thought that the reliability for the circular coil is good enough to warrant its use in the evaluation of MEPs and in finding hotspots.

Because the facilitatory effects using a focal coil were similar to those with a circular coil, we applied the same rMT found by the circular coil to define the rTMS intensity [20]. Our protocol is outside the safety guideline that Rossi et al. [21] recommended. No side effects such as seizure, syncope, or headache were noted.

The strength of the current study lies in the examination of the ability of multiple variables recorded before rTMS to predict behavioral effects in subacute stroke patients. Previously, very few studies were conducted to identify what characteristics determine individual responses to rTMS. Also, most prior studies focused on chronic or subcortical strokes [3,5,22-26]. We applied rTMS in the subacute period and included both subcortical and cortical stroke patients.

This study has several limitations. One was the absence of novel imaging techniques, such as diffusion-tensor imaging and magnetization transfer imaging that would allow for a better estimation of white matter integrity and connectivity, which might play an important role in rTMS treatment. Second, the study was conducted with a small number of patients, so a further larger-scale study is needed for better generalizability. Third, we only measured functional improvements two weeks after rTMS. This fact encourages future studies to investigate predictive variables of the long-term effects of rTMS on upper extremity motor recovery.

In conclusion, the results of this study suggest that rTMS may have a greater effect on upper extremity motor recovery in stroke patients who have a MEP response, with exclusively subcortical stroke, mild paresis, and good functional status. Even though these effects do not show immediately after rTMS, when a patient has a MEP response, with exclusively subcortical stroke and good cognitive function, we could expect delayed effects for up to two weeks. Applying rTMS early would have additional positive effects.

\section{CONFLICT OF INTEREST}

No potential conflict of interest relevant to this article was reported.

\section{REFERENCES}

1. Corti M, Patten C, Triggs W. Repetitive transcranial magnetic stimulation of motor cortex after stroke: a focused review. Am J Phys Med Rehabil 2012;91:254-70.

2. Hsu WY, Cheng CH, Liao KK, Lee IH, Lin YY. Effects of repetitive transcranial magnetic stimulation on motor functions in patients with stroke: a meta-analysis. Stroke 2012;43:1849-57.

3. Ameli M, Grefkes C, Kemper F, Riegg FP, Rehme AK, Karbe H, et al. Differential effects of high-frequency repetitive transcranial magnetic stimulation over ipsilesional primary motor cortex in cortical and subcortical middle cerebral artery stroke. Ann Neurol 2009;66:298-309.

4. Chang WH, Kim YH, Bang OY, Kim ST, Park YH, Lee PK. Long-term effects of rTMS on motor recovery in patients after subacute stroke. J Rehabil Med 2010;42: 758-64.

5. Kim YH, You SH, Ko MH, Park JW, Lee KH, Jang SH, et al. Repetitive transcranial magnetic stimulationinduced corticomotor excitability and associated motor skill acquisition in chronic stroke. Stroke 2006;37: 1471-6.

6. Khedr EM, Etraby AE, Hemeda M, Nasef AM, Razek AA. Long-term effect of repetitive transcranial magnetic stimulation on motor function recovery after acute ischemic stroke. Acta Neurol Scand 2010;121:30-7.

7. Hoyer EH, Celnik PA. Understanding and enhancing motor recovery after stroke using transcranial magnetic stimulation. Restor Neurol Neurosci 2011;29: 395-409.

8. Emara T, El Nahas N, Elkader HA, Ashour S, El Etrebi A. MRI can predict the response to therapeutic repetitive transcranial magnetic stimulation (rTMS) in stroke patients. J Vasc Interv Neurol 2009;2:163-8.

9. Miyamoto S, Kondo T, Suzukamo Y, Michimata A, Izu- 
mi S. Reliability and validity of the Manual Function Test in patients with stroke. Am J Phys Med Rehabil 2009;88:247-55.

10. Kohler F, Dickson H, Redmond H, Estell J, Connolly C. Agreement of functional independence measure item scores in patients transferred from one rehabilitation setting to another. Eur J Phys Rehabil Med 2009; 45:479-85.

11. Loewen SC, Anderson BA. Predictors of stroke outcome using objective measurement scales. Stroke 1990; 21:78-81.

12. Jung HY, Park BK, Shin HS, Kang YK, Pyun SB, Paik NJ, et al. Development of the Korean Version of Modified Barthel Index (K-MBI): Multi-center Study for Subjects with Stroke. J Korean Acad Rehabil Med 2007;31:283-97.

13. Norman GR, Sloan JA, Wyrwich KW. Interpretation of changes in health-related quality of life: the remarkable universality of half a standard deviation. Med Care 2003;41:582-92.

14. Talelli P, Greenwood RJ, Rothwell JC. Arm function after stroke: neurophysiological correlates and recovery mechanisms assessed by transcranial magnetic stimulation. Clin Neurophysiol 2006;117:1641-59.

15. Nowak DA, Grefkes C, Ameli M, Fink GR. Interhemispheric competition after stroke: brain stimulation to enhance recovery of function of the affected hand. Neurorehabil Neural Repair 2009;23:641-56.

16. Lefaucheur JP. Stroke recovery can be enhanced by using repetitive transcranial magnetic stimulation (rTMS). Neurophysiol Clin 2006;36:105-15.

17. Takeuchi N, Tada T, Toshima M, Matsuo Y, Ikoma K. Repetitive transcranial magnetic stimulation over bilateral hemispheres enhances motor function and training effect of paretic hand in patients after stroke. J Rehabil Med 2009;41:1049-54.

18. Fleming MK, Sorinola IO, Newham DJ, RobertsLewis SF, Bergmann JH. The effect of coil type and navigation on the reliability of transcranial magnetic stimulation. IEEE Trans Neural Syst Rehabil Eng 2012; 20:617-25.
19. Malcolm MP, Triggs WJ, Light KE, Shechtman O, Khandekar G, Gonzalez Rothi LJ. Reliability of motor cortex transcranial magnetic stimulation in four muscle representations. Clin Neurophysiol 2006;117:103746.

20. Shimizu T, Filippi MM, Palmieri MG, Oliveri M, Vernieri F, Pasqualetti P, et al. Modulation of intracortical excitability for different muscles in the upper extremity: paired magnetic stimulation study with focal versus non-focal coils. Clin Neurophysiol 1999;110:57581.

21. Rossi S, Hallett M, Rossini PM, Pascual-Leone A; Safety of TMS Consensus Group. Safety, ethical considerations, and application guidelines for the use of transcranial magnetic stimulation in clinical practice and research. Clin Neurophysiol 2009;120:2008-39.

22. Yozbatiran N, Alonso-Alonso M, See J, Demirtas-Tatlidede A, Luu D, Motiwala RR, et al. Safety and behavioral effects of high-frequency repetitive transcranial magnetic stimulation in stroke. Stroke 2009;40:309-12.

23. Fregni F, Boggio PS, Valle AC, Rocha RR, Duarte J, Ferreira MJ, et al. A sham-controlled trial of a 5-day course of repetitive transcranial magnetic stimulation of the unaffected hemisphere in stroke patients. Stroke 2006;37:2115-22.

24. Dafotakis M, Grefkes C, Eickhoff SB, Karbe H, Fink GR, Nowak DA. Effects of rTMS on grip force control following subcortical stroke. Exp Neurol 2008;211:40712.

25. Nowak DA, Grefkes C, Dafotakis M, Eickhoff S, Kust J, Karbe H, et al. Effects of low-frequency repetitive transcranial magnetic stimulation of the contralesional primary motor cortex on movement kinematics and neural activity in subcortical stroke. Arch Neurol 2008; 65:741-7.

26. Takeuchi N, Tada T, Toshima M, Chuma T, Matsuo Y, Ikoma K. Inhibition of the unaffected motor cortex by $1 \mathrm{~Hz}$ repetitive transcranical magnetic stimulation enhances motor performance and training effect of the paretic hand in patients with chronic stroke. J Rehabil Med 2008;40:298-303. 